\title{
Review on the Relationship between Feedback Environment and
}

\author{
Performance Appraisal ( 2004-2015) \\ XiaoYu Shi ${ }^{*}$ \\ School of Economics and Management, Nanjing University of Science and Technology, \\ China \\ *Corresponding author: XiaoYu Shi, Master, 422205817@qq.com
}

\begin{abstract}
Levy and Williams (2004) established a framework about the social context of performance appraisal. Feedback environment/feedback culture refers to one of the most significant process proximal variables. In the meanwhile, the majority of researchers have paid attention to the relationship between feedback environment and performance appraisal. Firstly, the paper reviews the concept and measurement of feedback environment. Furthermore, it focuses on reviewing research about feedback environment, feedback-seeking behaviour, and other active behaviours. Finally, some suggestions are proposed for the future research.
\end{abstract}

Key words: feedback environment/culture; performance appraisal; review

\section{Introduction}

As a crucial procedure of the performance appraisal, the performance feedback runs through all the aspects of performance management. Specifically, it can influence people's behavior, help individuals adjust their intuition, self-evaluation as well as self-management, and keep employees towards a predetermined target of organizations. Ultimately, the performance feedback can contribute to performance improvement. Thus, performance feedback is valuable organizational resource.

As argued by London (2002), the feedback culture can be linked to the other elements of performance management cycle ${ }^{1}$. In other words, feedback culture is of great significance for the way how feedback is sought, processed, accepted, perceived, used, and reacted to. Moreover, the entire feedback process is influenced by the feedback culture. Hence, the study of feedback culture should play a vital role in shedding light on performance management process ${ }^{1}$. Additionally, Levy and Williams (2004) developed a model and conducted a systematic review of the effects of the social context on the appraisal process ${ }^{2}$. Meanwhile, they conducted an analytical framework consisting of distal factors, and proximal variables. Proximal variables can be categorized as process proximal variables and structural proximal variables. To be specific, the process proximal variables are composed by rater issues, rate issues, leader-member dyadic issues, and group dynamics. Group dynamics contain three 
components: including politics and impression management, the feedback environment or culture experienced by organizational employees, work group or team processes ${ }^{2}$.

When Levy and Williams carried out literature review about feedback environment, there are very few researches in this area. However, over the past decades, some scholars have conducted meaningful research in relevant areas. As pointed out by some scholars, the supportive feedback environment can repair the damage of the performance system ${ }^{3}$. Since the feedback environment is the vital factor that will influence the performance appraisal, this paper mainly focuses on the impact of feedback environment on performance appraisal over the past decade, which can promote the improvement of employee performance.

\section{The concept and measurement of feedback environment}

\subsection{The concept of feedback environment}

In the past, feedback environment is defined as the information of job performance that employees perceive as being available to them (Herold \& Parsons, 1985) ${ }^{4}$. Subsequently, scholars define it as the amount and availability of positive and negative feedback from different sources. However, this is not the concept of feedback culture, as argued by London (2003). Furthermore, Steelman et al. (2004) stated that the feedback environment is the social context factors related to formal feedback process concerning supervisor -subordinate or peer-to-peer, rather than the feedback process which is part of the formal performance appraisal $^{5}$. As mentioned by him, the feedback environment can offer support to feedback. Also, it can ensure that the feedback is a worthwhile goal to pursue. What's more, he highlighted the need for additional training and development.

\subsection{The measurement of feedback environment}

Based on the clear definition of feedback environment, Steelman et al. (2004) also contributed to the development of the Feedback Environment Scale. The facets of the feedback environment include the supervisor source and coworker source. Both of the sources contain seven specific facets: (1) source credibility, (2) feedback quality, (3) feedback delivery, (4) frequency of favorable feedback, (5) frequency of unfavorable feedback, (6) source availability, (7) promoting feedback seeking ${ }^{5}$.

Source credibility. Source credibility refers to the expertise and trustworthiness of the feedback source. To be specific, the expertise includes knowledge about feedback recipient's job requirements, knowledge about recipient's job performance as well as the ability to judge job performance accurately. Trustworthiness means whether an individual believes that the feedback source provides accurate performance information. Scholars argued that feedback information provided by credible sources poses greater impact on the recipient's behaviors (Albright \& Levy, 1995; Ilgen et al., 1979; Makiney \& Levy, 1998) ${ }^{6-8}$.

Feedback quality refers to consistency and usefulness. High-quality feedback keeps consistent during a period of time. Compared with low-quality feedback, it is perceived being 
more effective. Low-quality feedback may vary with the feedback source's mood, observational opportunity, or how much recipient loves the feedback target. All in all, the feedback quality will affect the decision and reaction made by the recipient ${ }^{7}$.

Feedback delivery describes the recipient's perception of the source's intention. The more thoughtful the feedback source is, the more likely an individual accepts and responds to the feedback. As proved by most of researchers, thoughtful and considerate feedback has positive relationship with the perception of feedback atmosphere, outcomes of the performance feedback, and satisfaction with the feedback (Ilgen, Peterson, Martin, Boeschen, 1981) ${ }^{9}$.

Favorable and unfavorable feedback refers to the perception frequency of positive and negative feedback respectively, such as praise or complaint from supervisors or colleagues.

Source availability. In terms of source availability, the formal performance evaluation usually occurs once a year. However, individuals need to gain more useful information during the informal ordinary communications. The availability of information from supervisors or colleagues can be defined as the perceived amount of contact a staff has with his supervisor or colleagues and the convenience with which the feedback can be obtained ${ }^{10}$.

Feedback seeking behavior is one of the most dominant themes that have been studied by scholars in the past several years. Feedback seeking promotion refers to the extent to which the environment is supportive or unsupportive of feedback seeking behaviors (Williams, Miller, Steelman, \& Levy, 1999). According to the finding of the researchers, the most important determinant of feedback seeking behavior is the extent to which supervisor promotes the feedback seeking behavior ${ }^{11}$.

In a word, feedback environment can be defined as the overall workplace supportive feedback perceived by employees. The construct has multiple levels, which is composed by seven facets. Based on these facets, Steelman et al. (2004) developed Feedback Environment Scale based on these facets, and examined the construct validity, internal consistency, test-retest reliability, distinction validity and so on ${ }^{5}$.

\section{Feedback environment and performance appraisal}

\subsection{Feedback environment and feedback seeking behaviour}

Feedback seeking behavior means that the individual makes a conscious effort to obtain some valuable information, so as to judge whether the behavior is correct or whether the procedure is proper (Ashford, 1983) ${ }^{10}$. The biggest difference between the feedback seeking behavior and traditional feedback view is that the recipient of the feedback can be described as active person who owns self-regulated learning ability. Since the construct was put forward, feedback seeking behavior has raised the majority of scholars' attention. Meanwhile, there are a lot of empirical researches about the antecedents and consequences of feedback seeking behavior $^{12}$. De Stobbeleir, Ashford, and Buyens (2011) argued that the feedback seeking behavior can improve their task performance ${ }^{13}$. Similarly, Hays and Williams (2011) summarized that feedback seeking behaviors can reduce the uncertainty, which will help to 
improve job performance and a positive attitude ${ }^{14}$. Because of the positive consequence, it is meaningful to study the antecedent variables of the feedback seeking behaviors.

As demonstrated by multiple studies, the feedback environment perception has positive impact on informal feedback seeking behavior. Furthermore, Williams, Miller, Steelman, and Levy(1999) argued that the feedback seeking behaviors will be promoted when employees feel comfortable or perceive being encouraged, rewarded ${ }^{11}$. Additionally, Whitaker, Dahling, Levy (2007) conducted a study about the relationship between feedback environment and work performance ${ }^{15}$, and their study collected 170 group data about supervisor-subordinate. As shown by the results, both supportive supervisor feedback environments and supportive colleague feedback environments can positively influence the feedback seeking behaviors. What's more, feedback seeking behaviors will make role task clear and lead to better performance ratings (including task performance and contextual performance). Particularly, a supportive supervisor environment can enhance the clarity of role task, without feedback seeking behaviors. Also, the feedback seeking behavior can pose direct impact on task performance without role clarity. In the supportive colleague environment, efforts about colleagues can negatively moderate the relationship between environments and feedback seeking behaviors.

\subsection{Feedback environment and other performance management seeking behaviour}

Also, the organizational performance management expects employees to show other behaviors, and this section will describe the feedback environment as well as the organizational desired behavior.

Rosen et al. (2006) conducted a model consisting of political perception, feedback environment, staff attitude, work performance ${ }^{16}$. Based on 150 group data concerning supervisor-subordinate, the study demonstrated that the supportive supervisors or colleagues feedback environment can enhance the performance by reducing employees' perception of the organization.

Moreover, Mulder et al. (2013) explored the perceived feedback quality ${ }^{17}$. In this paper, it emphasizes the significance of feedback quality. The perception of feedback quality is one of the paths, through which the environment can influence the other variables.

Pichler (2012) carried out a study about the relationship between the context of performance appraisal and appraisal reactions ${ }^{18}$. The study is composed by three different models: (1) Relationship quality, performance ratings, and appraisal participation are all regarded as independent predictors of appraisal reactions. (2) Relationship quality can influence the appraisal reactions through rating favorability and appraisal participation. (3) Relationship quality can not only indirectly influence appraisal reactions but also affect appraisal reactions through the rating favorability or appraisal participation.

$\mathrm{Li}$ et al. (2011) conducted a study about the a new concept-developmental feedback and focused on the role of developmental feedback and proactive personality on newcomer 
performance and helping behavior ${ }^{19}$. The development feedback refers to the extent of useful and helpful information that organizational members can offer to the newcomers. Moreover, this information enables the new newcomers to learn, develop and obtain good job performance. As displayed by the results, the development feedback (supervisor / colleague) can promote the helpful behaviors of new comers in the workplace.

\section{Conclusion and future research}

In recent years, foreign scholars conducted a series of studies on feedback environment. However, the research on feedback environment is still limited. Indeed, domestic scholars make little research on the feedback environment. Multiple studies have proved the positive influence of feedback environment on the employees' attitudes and behaviors. Thus, further studies are meaningful.

1) Systematic study. The majority of researches just pay attention to a part of feedback environment, which is far from systematic. In recent years, the consequences of feedback environment are overlooked in recent years. Feedback quality, positive or negative feedback is different form of feedback environment. Indeed, focusing on a little part of the study may make researchers to ignore some key influences. Hence, the key influencing mechanism of feedback environment deserves deep study.

2) Measures to promote environmental feedback. The studies on the key factors which influence the formation of feedback environment are insufficient. There is no doubt that promoting the formation of supportive feedback environment will certainly facilitate the organizational performance management. It is essential to strengthen research on feedback in the future.

\section{References}

1. M. London, J. W. Smither, Feedback orientation, feedback culture, and the longitudinal performance management process. Human Resource Management Review. 12 (2002) 81-100.

2. P. E. Levy, J. R. Williams, The social context of performance appraisal: A review and framework for the future. Journal of management. 30 (2004) 881-905.

3. J. J. Dahling, A. L. O’Malley, Supportive feedback environments can mend broken performance management systems. Industrial and Organizational Psychology. 4 (2011) 201-203.

4. D. M. Herold, C. K. Parsons, Assessing the Feedback Environment in Work Organizations: Development of the Job Feedback Survey. Journal of Applied Psychology. 70 (1985) 290-305.

5. L. A. Steelman, P. E. Levy, A. F. Snell, The feedback environment scale: Construct definition, measurement, and validation. Educational And Psychological Measurement. 64 (2004) 165-184. 
6. M. D. Albright, P. E. Levy, The Effects of Source Credibility and Performance Rating Discrepancy on Reactions to Multiple Raters 1. Journal of Applied Social Psychology. 25 (1995) 577-600.

7. D. R. Ilgen, C. D. Fisher, M. S. Taylor, Consequences of individual feedback on behavior in organizations. Journal of Applied Psychology. 64 (1979) 349-371.

8. J. D. Makiney, The Influence of Self-Ratings versus Peer Ratings on Supervisors Performance Judgments. Organizational Behavior \& Human Decision Processes. 74 (1998) 212-228.

9. D. R. Ilgen, R. B. Peterson, B. A. Martin, D. A. Boeschen, Supervisor and subordinate reactions to performance appraisal sessions. Organizational Behavior \& Human Performance. 28 (1981) 311-330.

10. S. J. Ashford, L. L. Cummings, Feedback as an individual resource: Personal strategies of creating information. Organizational Behavior \& Human Performance. 32 (1983) 370-398.

11. J. R. Williams, L. A. Steelman, C. E. Miller, P. E. Levy, Increasing feedback seeking in public contexts: It takes two (or more) to tango. Journal of Applied Psychology. 84(6) (1999) 969-976.

12. F. Anseel, A. S. Beatty, W. Shen, F. Lievens, P. R. Sackett, How are we doing after 30 years? A meta-analytic review of the antecedents and outcomes of feedback-seeking behavior. Journal of Management. 41 (2015) 318-348.

13. K. E. De Stobbeleir, S. J. Ashford, D. Buyens, Self-regulation of creativity at work: The role of feedback-seeking behavior in creative performance. Academy of Management Journal. 54 (2011) 811-831.

14. J. C. Hays, J. R. Williams, Testing multiple motives in feedback seeking: The interaction of instrumentality and self protection motives. Journal of Vocational Behavior. 79 (2011) 496-504.

15. B. G. Whitaker, J. J. Dahling, P. Levy, The development of a feedback environment and role clarity model of job performance. Journal of Management. 33 (2007) 570-591.

16. C. C. Rosen, P. E. Levy, R. J. Hall, Placing perceptions of politics in the context of the feedback environment, employee attitudes, and job performance. The Journal of applied psychology. 91 (2006) 211-220.

17. R. H. Mulder, A. D. Ellinger, R. H. Mulder, A. D. Ellinger, Perceptions of quality of feedback in organizations: Characteristics, determinants, outcomes of feedback, and possibilities for improvement: Introduction to a special issue. European Journal of Training and Development. 37 (2013) 4-23.

18. S. Pichler, The social context of performance appraisal and appraisal reactions: A meta-analysis. Human Resource Management. 51 (2012) 709-732.

19. N. Li, T. B. Harris, W. R. Boswell, Z. Xie, The role of organizational insiders' 
developmental feedback and proactive personality on newcomers' performance: an interactionist perspective. Journal of Applied Psychology. 96 (2011) 1317. 